\title{
NOUVELL
}

\section{Des organes sur puce au service de la cancérologie}

Annie Molla, Jean-Luc Coll
Institut pour l'avancée des biosciences (IAB), équipe «thérapie ciblée, diagnostic précoce et imagerie du cancer », Inserm U1209, CNRS UMR5309, Université Grenoble Alpes, allée des Alpes, 38700 Grenoble, France. annie.molla@univ-grenoble-alpes.fr
> Après des décennies à étudier les cellules cancéreuses humaines adhérant sur une surface plastique, la biologie cellulaire s'est progressivement complexifiée en intégrant la $3^{e}$ dimension. Des dépôts de matrice extracellulaire peuvent être réalisés par des imprimantes 3D reconstituant ainsi in vitro, des réseaux de fibres de collagène [1] $(\rightarrow)$. De nombreuses techniques de cultures cel- $\rightarrow$ Voir la Nouvelle de N. Elkahatib et G. Montagnac, $m / s$ $n^{\circ}$ 6-7, juin-juillet 2018, page 522 lulaires en 3D ont également été décrites ces quinze dernières années. Les cellules sont soit déposées sur un lit de matrice extracellulaire (souvent du matrigel ${ }^{T M}$ ), soit cultivées dans des conditions empêchant leur adhérence (puits de faible adhérence, lit d'agarose ou en goutte pendante). Ces modèles reproduisent les interactions cellule-cellule et seule la couche externe de cellules est en contact constant avec les nutriments. Une zone hypoxique se crée ainsi progressivement au centre de l'agrégat cellulaire, nommé organoïde ou sphéroïde (Figure 1). II a été montré que les récepteurs de surface, comme l'EGFR (epithelial growth factor receptor), étaient plus faiblement exprimés dans les sphéroïdes, comparativement à ce qui est observé avec les mêmes cellules cultivées en 2 dimensions (2D) (Figure 1). La signalisation intracellulaire y est donc différente.

Un des avantages des cultures 3D est également de pouvoir introduire au sein des sphéroïdes des cellules annexes qui sont présentes dans les tumeurs, comme les fibroblastes ou les cellules immunitaires. Ainsi, l'ajout de fibroblastes de patients à des cellules de mélanome cultivées en 3D modifie les voies de prolifération des cellules cancéreuses avec la réactivation de la voie ERK (extracellular signal-regulated kinase) via Fak (focal adhesion kinase) au détriment de BRAF (v-raf murine sarcoma viral oncogene homo$\log$ B1) [2]. Toutefois, ces cultures en suspension présentent des défauts de cytodiérèse et ne peuvent donc totalement rendre compte de la croissance des tumeurs [3]. Ces cultures ne reproduisent également pas les interactions et les échanges entre la tumeur, les tissus voisins et le système vasculaire environnant, qui sont des contributeurs importants à sa progression et donc essentiels à la lutte contre le cancer. Pour pallier ces défauts et définir des modèles thérapeutiques plus prédictifs, une nouvelle technologie se développe: il s'agit d'organes sur puce ${ }^{l}$ (OSP) intégrés dans un système de microfluidique. Le groupe de Donald Ingber, précurseur dans cette technologie, a publié récemment une revue dans Nature Rev Cancer [4], qui décrit les systèmes existants et leurs potentialités. Dans cette nouvelle, nous analyserons les OSP présentés dans cette revue en centrant le propos sur le cancer du poumon $[4,5]$.

\section{Description de la puce poumon}

Ces dispositifs miniaturisés utilisent la technologie de la microfluidique et de la culture cellulaire pour reproduire l'environnement complexe d'une tumeur au sein d'un organe. Les cellules tumorales reposent sur une monocouche de cellules épithéliales de poumon non transformées, elle-même en contact avec une matrice extracellulaire (MEC) enrichie en fibroblastes normaux et perfusée par du milieu de culture. La MEC est déposée sur une membrane synthétique poreuse qui assure l'interface avec le compartiment basal (Figure 2) [5, 6]. Ce tissu reconstitué est, dans sa partie basale,

${ }^{1}$ Elles sont appelées «puces» car, à l'origine, leur élaboration est adaptée des méthodes de microfabrication des composants d'ordinateurs. 
Culture 2D
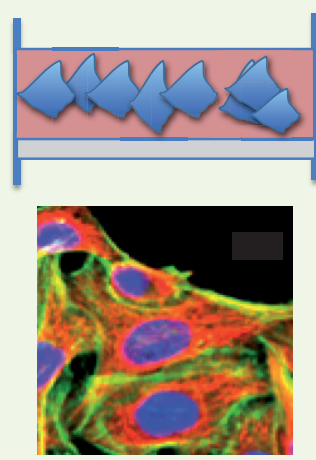

$2-4$ jours

Mono-culture
3D

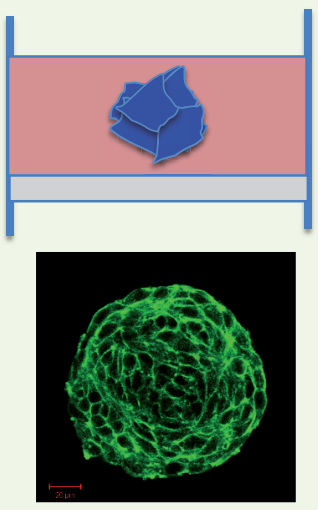

Plusieurs semaines

Ajout de cellules annexes

Organe sur puce

Régulation de la circulation

d'air-Contrainte cyclique ou continue

Perfusion ECM - cellules stromales

Perfusion cellules endothéliales

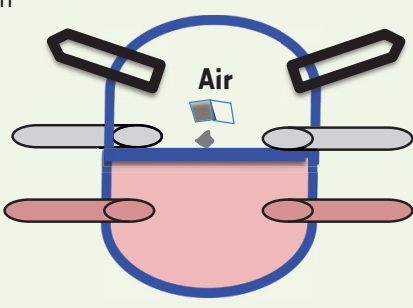

Plusieurs semaines

Reproduit les interactions tissulaires et les contraintes mécaniques Expression des récepteurs EGFR modulée par le flux d'air

Expression des récepteurs EGFR

différente entre $2 \mathrm{D}$ et $3 \mathrm{D}$

Prédiction de l'efficacité de drogues en oncologie

Figure 1. Complexification des cultures de cellules cancéreuses. À l’origine, les cellules cancéreuses étaient cultivées sur supports plastiques. Des cultures en 3-dimensions ont été progressivement développées et ont été complexifiées avec des cellules annexes (fibroblastes ou cellules immunitaires). Les cellules cultivées en 3D ont un taux de renouvellement plus faible que les cellules des cultures en 2D et peuvent être étudiées à long terme. Plus récemment, des organes sur puce (OSP), intégrés dans un système de microfluidique, ont été élaborés. Les OSP reproduisent les contacts entre le tissu sain, la tumeur et son environnement, et intègrent les données chimiques et mécaniques. Ces cultures peuvent être maintenues à long terme. L'expression des récepteurs de surface comme l'EGFR (epithelial growth factor receptor) est modulée par les conditions de culture, soulignant l'intérêt de ces modèles pour évaluer l'efficacité de drogues en oncologie.

en contact avec un tapis de cellules endothéliales perfusées et, dans sa partie apicale, au contact avec l'air. Le compartiment principal est situé entre deux chambres creuses au travers desquelles une aspiration cyclique peut être appliquée, imitant ainsi les signaux mécaniques des organes et, dans le cas du poumon, reproduisant les interfaces air-liquide et la force mécanique de la respiration $[5,6]$. Ainsi, I'OSP poumon respire!

\section{Intérêt de ces dispositifs}

Ces dispositifs permettent d'analyser la plupart des étapes de progression du cancer : la croissance tumorale et son expansion, l'angiogenèse, la transition épithélio-mésenchymateuse (EMT), la migration et l'invasion d'une cellule tumorale et les étapes métastatiques [4].

Dans I'OSP poumon, la croissance des cellules de cancer du poumon humain non à petites cellules (CPNPC) dépend entièrement de facteurs micro-environnementaux locaux produits par les cellules épithéliales alvéolaires normales et par les cellules endothéliales du poumon qui ont été interfacées à travers la membrane poreuse (Figure 2). Ces cellules de cancer du poumon restent au repos pendant environ 12 jours de culture sur la puce avant d'initier une croissance logarithmique avec un temps de doublement d'environ 40 heures (dans une culture 2D, il est d'environ $30 \mathrm{~h}$ ). Cette étape de dormance tumorale mime en partie la maladie résiduelle et ainsi les OSP permettent de tester des stratégies pour la combattre [5].

La proximité entre les cellules stromales et les cellules cancéreuses facilite les échanges de facteurs solubles. II est ainsi possible d'étudier la progression tumorale. Le milieu conditionné de l'épithélium alvéolaire sain s'avère suffisant pour favoriser la prolifération des cellules cancéreuses, mais les facteurs sécrétés par les cellules endothélialles réduisent cette croissance tumorale. Après 14 jours de culture, des cellules tumorales traversent la membrane séparant l'épithélium de l'endothélium. Elles s'accumulent dans le canal vasculaire où elles prolifèrent. Cette extravasation est moindre quand l'OSP est soumis à des mouvements respiratoires [5]. 


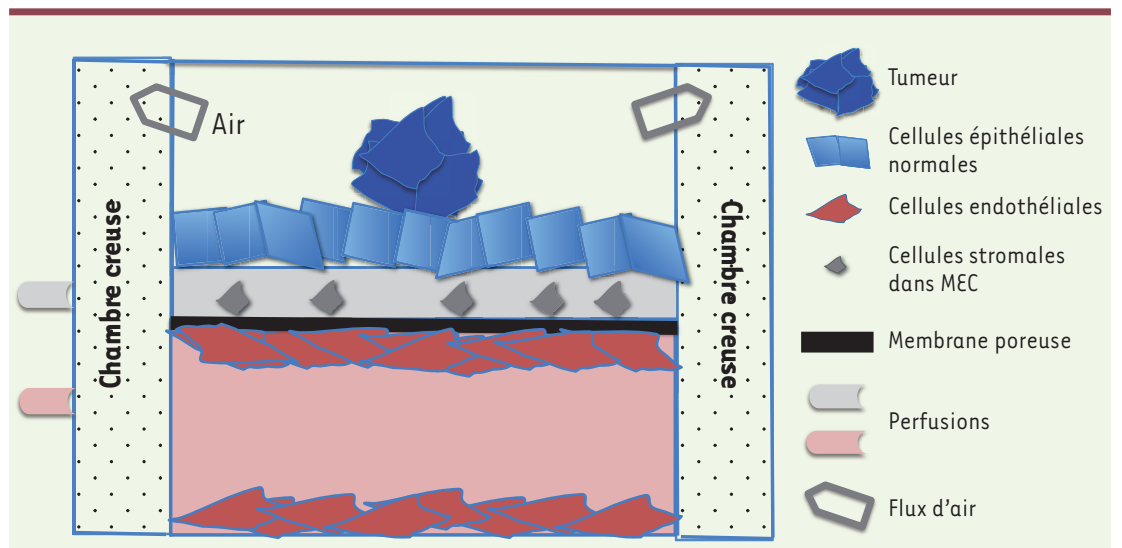

Effets de la respiration

- Réduction de la croissance et de l'invasion tumorales

- Diminution de la phosphorylation des récepteurs de l'EGF

- Modification de la réponse aux drogues

Figure 2. La puce poumon. Les cellules tumorales reposent sur une monocouche de cellules épithéliales de poumon non transformées, qui est au contact d'une matrice extracellulaire (MEC) enrichie en fibroblastes normaux. La MEC est déposée sur une membrane synthétique poreuse qui assure l'interface avec le compartiment basal, lui-même constitué d'un tapis de cellules endothéliales de poumon. L'ensemble est perfusé et la partie apicale est au contact avec l'air. Le compartiment principal est situé entre deux chambres creuses au travers desquelles une aspiration cyclique peut être appliquée, qui mime la force mécanique de la respiration. Les modifications induites par la force mécanique sont indiquées. EGF : epithelial growth factor.

Cette technologie a également dévoilé les contributions des différents types cellulaires pour la migration et l'envahissement des cellules tumorales. L'analyse de la boucle paracrine entre les cellules humaines d'adénocarcinome pulmonaire CLI- 0 et les fibroblastes de poumon MRC- 5 révèle que le TGF- $\beta$ (transforming growth factor- $\beta$ ), sécrété par les cellules cancéreuses, stimule la transformation des fibroblastes en myofibroblastes qui, en retour, favorisent la migration des cellules cancéreuses [7]. Des études in vivo antérieures avaient révélé que les cytokines IL-6 (interleukin-6) et IL-8, ainsi que le VEGF (vascular endothelial growth factor) pouvaient être des indicateurs pronostiques cliniquement importants de la croissance du cancer du poumon $[8,9]$. L'OSP dispose d'un écoulement de fluide dans le canal vasculaire, il est donc possible d'analyser les composants sécrétés en recueillant les effluents au fil du temps dans ce compartiment basal. Les études utilisant I'OSP pou-

d'IL-6 et de VEGF étaient effectivement élevées dans les effluents, mais que les taux d'IL-8 diminuaient, et cela quelque soit la force mécanique appliquée au dispositif. Lorsque les cellules tumorales du poumon sont traitées par le rociletinib, un inhibiteur de tyrosine kinase (ITK) de troisième génération, une réduction considérable des taux d'IL-6 et d'IL-8 est observée parallèlement à une augmentation des taux de VEGF. Les niveaux de suppression des deux cytokines induits par l'inhibiteur sont significativement plus importants dans les puces exposées aux mouvements physiologiques de respiration [5]. Une telle réduction des taux d'IL-8 au cours des traitements par les ITK des cancers du poumon a également été rapportée dans les essais cliniques chez I'homme [8, 9].

Quand une contrainte cyclique mimant les mouvements respiratoires physiologiques est exercée sur le capillaire alvéolaire, la croissance des cellules cancéreuses est inhibée d'environ $50 \%$. Toutefois, la contrainte mécanique induit une baisse d'expression de l'EGFR et de sa phosphorylation et conduit in fine à une résistance accrue au rociletinib [5]. Cette absence de réponse aux ITK s'explique par une modification de la signalisation intracellulaire et par la surexpression de la kinase $c-$ Met $^{2}[10]$ $(\rightarrow)$. $(\rightarrow)$ Voir la Synthèse de R. Montagne et al., $\mathrm{m} / \mathrm{s} \mathrm{n}^{\circ} 10$, octobre 2014, page 864
Ce même mécanisme de résistance est décrit chez les patients atteints de CPNPC, confirmant les potentialités des OSP pour prédire les réponses aux thérapies [11].

Ces réponses, qui n'auraient pas pu être évaluées sans l'utilisation de cette technologie, pourraient expliquer des situations physiopathologiques. Des cancers du poumon, qui se développent rapidement et perturbent le mouvement alvéolaire, répondent efficacement à un traitement qui induit pourtant des résistances chez d'autres patients avec une maladie résiduelle minimale et une fonctionnalité pulmonaire normale $[4,5]$.

\section{Généralisation des OSP \\ et de leurs applications}

De nombreux micro-dispositifs OSP qui reproduisent les propriétés fonctionnelles et mécaniques de divers organes, comme les tubules rénaux, l'intestin grêle, le foie, la moelle osseuse et la barrière hématoencéphalique ont été créés [4]. Certains sont constitués non plus de cellules isolées, mais de «copeaux» d'organe et sont produits en série, ce qui augmente le champ de leurs applications. Les OSP permettent d'évaluer les réponses aux drogues dans des conditions presque physiologiques puisque ces puces peuvent être perfusées par du sang pendant quelques heures. Il est également envisageable de suivre l'impact sur ces cellules de pathogènes ou d'agressions environnemen-

${ }^{2}$ Le récepteur à activité tyrosine kinase Met et son ligand I'HGF (hepatocyte growth factor)/SF (scatter factor) sont des inducteurs potentiels de la transformation et de la prolifération cellulaires. 
tales (rayonnement, pesticides, fumée de cigarette, etc.). Ces OSP sont un élégant modèle pour étudier l'environnement tumoral, mais peuvent également mimer d'autres situations pathologiques comme l'œdème pulmonaire et la thrombose, l'asthme ou la maladie pulmonaire obstructive chronique (BPCO) [4].

Les OSP ouvrent donc de nouvelles perspectives pour le développement de médicaments et de stratégies thérapeutiques innovantes. Ces dispositifs pourront à terme permettre l'évaluation de thérapies ciblées sur des biopsies. Des dispositifs commerciaux existent, mais leur mise en œuvre est encore complexe. La plupart d'entre eux sont réalisés avec des polymères flexibles, comme le polydiméthylsiloxane (PDMS), dans lesquels des micro-canaux sont creusés. II semble que le PDMS puisse absorber certains facteurs et ait un effet sur l'homéostasie du système. Le choix des milieux de perfusion est également encore très empirique et demandera de longues mises au point. L'utilisation de ces dispositifs en médecine personnalisée pour prédire les réponses thérapeutiques est donc évoquée, mais ces thérapies seront encore hypothétiques tant que ces verrous technologiques ne seront pas levés.

Les OSP, par leur versatilité, leur mimétisme des situations physiopathologiques, à la fois chimiques (gradient de soluté) et mécanique (poumon, intestin, etc.), représentent néanmoins un pas de géant dans la recherche en oncologie. $\diamond$ Organs-on-chips dedicated to oncology

\section{LIENS D'INTÉRÊT}

Les auteurs déclarent n'avoir aucun lien d'intérêt concernant les données publiées dans cet article.

\section{RÉFÉRENCES}

1. Elkahatib N, Montagnac G. Une endocytose frustrée pour une migration accomplie Med Sci (Paris) 2018; $34: 522-4$.

2. Hirata $\varepsilon$, Girotti MR, Viros $A$, et al. Intravital imaging reveals how BRAF inhibition generates drug-tolerant microenvironments with high integrin $\beta 1 /$ FAK signaling. Cancer Cell $2015 ; 27: 574-88$.
3. Molla A, Couvet M, Coll JL. Unsuccessful mitosis in multicellular tumour spheroids. Oncotarget 2017 ; 8 : 28769-84.

4. Sontheimer-Phelps A, Hassell BA, Ingber DE. Modelling cancer in microfluidic human organs- on-chips. Nat Rev Cancer 2019 ; $19: 65-81$.

5. Hassell BA, Goyal G, Lee $\varepsilon$, et al. Human organ chip models recapitulate orthotopic lung cancer growth, therapeutic responses, and tumor dormancy in vitro. Cell Rep 2017 ; 21 : 508-16.

6. Benam KH, Villenave R, Lucchesi C, et al. Small airway-on-a-chip enables analysis of human lung inflammation and drug responses in vitro. Nat Methods $2016 ; 13: 151-7$

7. Hsu TH, Xiao JL, Tsao YW, et al. Analysis of the paracrine loop between cancer cells and fibroblasts using a microfluidic chip. Lab Chip 2011 ; 11 : 1808 14.

8. Enewold L, Mechanic LE, Bowman ED, et al. Serum concentrations of cytokines and lung cancer survival in African Americans and Caucasians. Cancer Epidemiol Biomarkers Prev 2009 ; 18 : 215-22.

9. Umekawa K, Kimura T, Kudoh S, et al. Plasma RANTES, IL-10, and IL-8 levels in non-small-cell lung cancer patients treated with EGFR-TKIs. BMC Res Notes 2013 $6: 139$

10. Montagne R, Furlan A, Kherrouche Z, Tulasne D. Le récepteur Met fête ses 30 ans : de la découverte d'un oncogène au développement de thérapies ciblées. Med Sci (Paris) $2014 ; 30: 864-73$.

11. Chabon JJ, Simmons AD, Lovejoy AF, et al. Circulating tumour DNA profiling reveals heterogeneity of EGFR inhibitor resistance mechanisms in lung cancer patients. Nat Commun $2016 ; 7: 11815$.

P ossédées du malin au Moyen-Âge, les sorcières hystériques sont vouées au bûcher. Enfermées au xvII siècle, maltraitées, elles rejoignent la Cour des Miracles de l'Hospice de la Vieillesse-Femmes à la Salpêtrière... Jusqu'à ce que le Dr JeanMartin Charcot (1825-1893) mène le combat qui transforme l'ancien hospice en hôpital : l'École de la Salpêtrière de

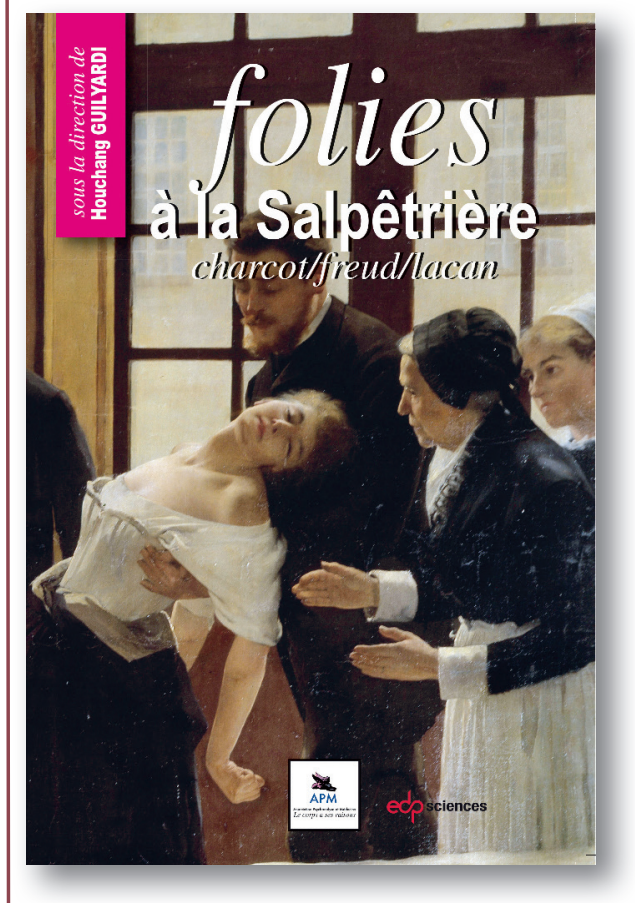

ISBN : 978-2-7598-1268-4
240 pages

$20 €$ Paris est née, qui devient lieu de recherche, d'enseignement et de soins, de renommée internationale.

Jean Martin Charcot n'a pas bonne presse, et pourtant... Hystérie et folie traversent les siècles, prenant les formes de «l'air du temps ».

De l'utérus migrateur d'Hippocrate aux recherches neurologiques de Charcot. Du désir inconscient avec Freud à la jouissance du parlêtre chez Lacan... C'est à cette traversée historique et conceptuelle que nous convie cet ouvrage.
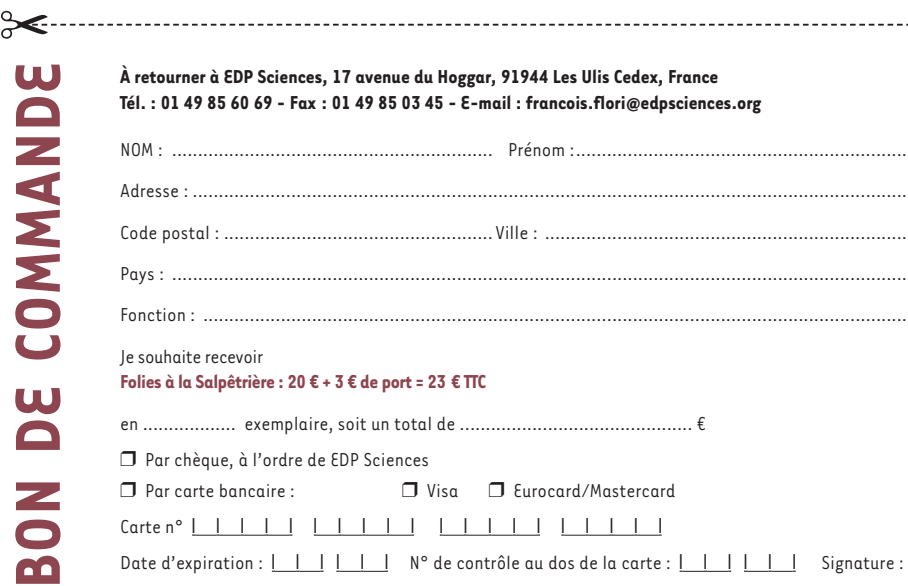